\title{
Incidence and Risk Factors Affecting Development of Perianal Fistulas after Drainage of Perianal Abscesses
}

\author{
Omar Mohammad Khalil Labib Al Ozaibi Hadiel M. Kaiyasah \\ Nour AIDeen Hejazi \\ General Surgery, Rashid Hospital, Dubai Health Authority, Dubai, United Arab Emirates
}

\section{Keywords}

Anal fistula · Perianal abscess $\cdot$ Risk factors · Surgery $\cdot$ United Arab Emirates

\begin{abstract}
Background: Perianal fistulas can present significant morbidity and detriment to a patient's quality of life. This study aimed to quantify the incidence of perianal fistulas and attempted to identify any risk factors for their development. Materials and Methods: This retrospective study reviewed all patients who underwent drainage of first-time perianal abscesses in Rashid Hospital from 2014 to 2018. Noncryptoglandular causes of fistula formation were excluded after which incidence and risk factors were investigated. Results and Discussion: A total of 445 records were reviewed of which 131 met the inclusion criteria (103 males and $28 \mathrm{fe}$ males). The median age of patients was 37 with ranges of $15-85$. There was no statistically significant finding relating to gender ( $p$ value $=1.00)$, smoking ( $p$ value $=1.00$ ), location of perianal abscess ( $p$ value $=0.347$ ), and postoperative antibiotics ( $p$ value $=0.694$ ). There was no statistically significant finding relating to patients below or above 40 ( $p$ value $=$ 0.846 ), and logistic regression testing revealed no statistical significance in age relating to development of perianal fistu-
\end{abstract}

$\begin{array}{ll}\text { karger@karger.com } & \text { ( ) 2021 The Author(s) } \\ \text { www.karger.com/dmj } & \text { Published by S. Karger AG, Basel } \\ & \text { This article is licensed under the Creative Commons Attribution- } \\ \text { Karger } & \begin{array}{l}\text { NonCommercial-NoDerivatives 4.0 International License (CC BY- } \\ \text { NC-ND) (http://www.karger.com/Services/OpenAccessLicense). } \\ \text { Usage and distribution for commercial purposes as well as any dis- } \\ \text { tribution of modified material requires written permission. }\end{array}\end{array}$

las. There was a $13.2 \%$ increased proportion of nondiabetics to develop perianal fistulas as compared to diabetics (32 vs. $19 \%)$, but it was not found to be statistically significant ( $p$ value $=0.236$ ). Conclusion: None of the risk factors studied were found to be statistically significant in the development of perianal fistulas after drainage of perianal abscesses and the incidence was found to be $29.8 \%$.

(c) 2021 The Author(s)

Published by S. Karger AG, Basel

\section{Introduction}

Perianal fistulas are a common sequelae to perianal abscesses. The most widely accepted theory for the pathogenesis of both is that of the cryptoglandular theory of Eisenhammer and Parks. The theory states that perianal abscesses are a result of the blockage of an intramuscular anal gland which subsequently cannot spontaneously drain into the anal canal $[1,2]$. Subsequently, even if there is spontaneous or iatrogenic drainage, it is hypothesized that the area of the abscess is a nidus for chronic infection which ultimately leads to fistula formation [3].

Perianal fistulas present a chronic significant morbidity to patients and may require recurrent surgical interventions which are detrimental to both financial and per- 
Fig. 1. Patient flowchart.

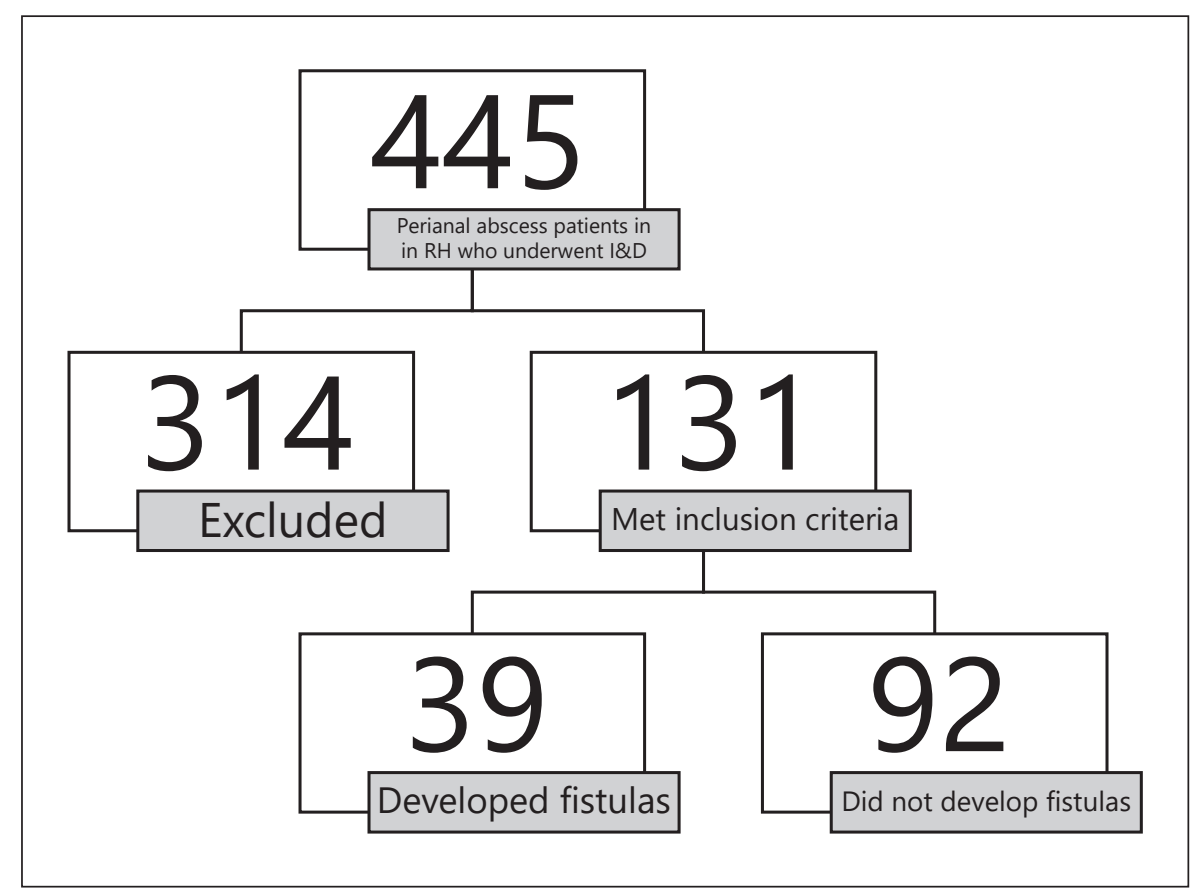

sonal quality of life to those affected. By identifying those who are at high risk for developing the fistulas, it would potentially be beneficial in various veins. Specifically, within the UAE, with rising incidence of diabetes and a lack of previous data on the multiple nationalities mixed demographic within the country, it would be helpful to see if the regional data differ at all from any similar study done elsewhere.

Within the current literature, there are various studies which attempted to answer the same question and overall, with mixed results [4-8]. The aim of this study is first, to shed light on etiology of the condition if any correlations to specific comorbids are highlighted. Second, it would help in targeting those who need further education and more vigilant follow-up to identify the condition early, which in turn, could aid in prevention by developing prophylactic interventions.

\section{Materials and Methods}

This study was performed in a retrospective cohort manner after ethical approval was obtained from the Dubai Scientific Research Ethics Committee. All patients who were diagnosed with a first-time perianal abscess in Rashid Hospital between 2014 and 2018 were analyzed. Patients who were under 16 were presenting with synchronous presence of anal fistula, had any documented presence of noncryptoglandular etiologies (malignancy, trauma, and Crohn's) were excluded. In addition, any patient found to have incomplete documentation on initial presentation or during follow-up was excluded.

The medical records of the patients were reviewed, and the information extracted from both their inpatient stay and outpatient follow-up included age, gender, smoking habit, diabetes, location of the abscess, and postoperative antibiotic use. Any information that was not immediately available from the records was ascertained using a telephone questionnaire after obtaining verbal consent from the patients. The primary outcome was the development of perianal fistula. All the information was collated and exported to SPSS (Version 23). Median and frequencies were reported as descriptive statistics. Mann-Whitney U test was used. The $\chi^{2} /$ Fisher's exact test was used for categorical variables, and the data were presented as numbers (percentages) as appropriate. A $p$ value of $<0.05$ was considered significant.

\section{Results}

A total of 445 patients were found to have undergone incision and after review, and 131 met the inclusion criteria and underwent further analysis (Fig. 1). The majority of patients were male $103(78 \%)$ and the median age of the patients was found to be 37, with a range of 15-85. $58 \%(n=77)$ of patients were below 40 years, $32 \%$ were found to be smokers $(n=43)$, and $20 \%(n=26)$ were diabetic. $63 \%(n=83)$ of patients received postoperative antibiotics. The majority of the abscesses were found to be perianal in location $(87 \%$ [ $n=114])$. The range of follow- 
up of patients, both in clinic and from telephonic interview, was between 6 months and 2-year post procedure with the majority of patients Table 1 .

The development of a perianal fistula was found in $29.8 \%$ of patients $(n=39)$. Univariate analysis (Table 2 ) revealed no significant difference between genders in the development of perianal fistulas. The same was found for both smoking and diabetes. Interestingly, diabetics were found to have a $13.2 \%$ less chance to develop perianal fistulas, although this number did not reach statistical significance. Age, tested as both categorical (above and below 40 years) and with logistic regression, showed no statistical significance in development of perianal fistulas.

\section{Discussion/Conclusion}

This study showed an almost $30 \%$ rate of development of fistulas. This is a similar rate to the some of the available literature $[4-6,9]$. It studied gender, age, smoking habit, diabetes, and postoperative antibiotics as risk factors for development and found none to be statistically significant. With regards to gender, while there was a predominance of male patients presenting with perianal abscesses, once they were drained, the chance of developing a fistula was almost identical between the 2 groups. This finding supports the Parks theory in that regardless of the mechanism which causes males to develop abscesses more frequently, once the abscess is drained, the remnant cavity for both genders is a nidus for infection which ultimately develops into a fistula.

Some studies have found younger age to be a significant risk factor $[4,5]$ in development of anal fistulas. Peaks of incidences vary, with some studies showing a peak between 20-30 [10], while others found it to be a decade later $[5,7]$. Smoking has been stipulated as an risk factor by multiple studies $[7,11]$ with the reasoning being that smoking is a pro-inflammatory behavior and would promote the inflammatory habitat which would allow for fistula formation post drainage. Our findings for diabetic patients were interesting in the sense that nondiabetics seemed to be more prone to developing perianal fistulas. These findings were echoed in other studies $[4,5]$ although these are not unanimous findings [7]. Keeping the Parks cryptoglandular theory in mind, it would be expected that antibiotics would play a protective role in development of fistulas. While the literature does show trends of this being the case $[12,13]$ and meta-analysis of the data showed the same [14], our data did not follow the same trend. This could be due to there being no standard-

Risk Factors for Development of Perianal Fistulas
Table 1. Patient demographic table

\begin{tabular}{lc}
\hline & Patients, $n(\%), n=131$ \\
\hline Gender & \\
$\quad$ Male & $103(79)$ \\
$\quad$ Female & $28(21)$ \\
Age & \\
$\quad<40$ & $77(59)$ \\
$\quad>40$ & $54(41)$ \\
Smokers & $43(33)$ \\
Nonsmokers & $88(67)$ \\
Diabetics & $26(20)$ \\
Nondiabetics & $105(80)$ \\
Postoperative antibiotics & $83(63)$ \\
No postoperative antibiotics & $48(37)$ \\
Location of abscess & \\
$\quad$ Intersphincteric & $6(5)$ \\
$\quad$ Ischiorectal & $10(8)$ \\
$\quad$ Perianal & $114(87)$ \\
$\quad$ Supralevator & $1(0.8)$ \\
\hline
\end{tabular}

Table 2. Univariate analysis risk factors

\begin{tabular}{lrl}
\hline & $\begin{array}{c}\text { Development of } \\
\text { fistula }(\%), n=39\end{array}$ & $\begin{array}{c}p \text { value } \\
(<0.05)\end{array}$ \\
\hline $\begin{array}{l}\text { Gender } \\
\quad \text { Male }\end{array}$ & $31(30)$ & \\
$\quad$ Female & $8(29)$ & \\
Age & & \\
$\quad<40$ & $22(29)$ & 0.846 \\
$\quad>40$ & $17(32)$ & 1 \\
Smokers & $13(30)$ & \\
Nonsmokers & $26(30)$ & 0.236 \\
Diabetics & $5(19)$ & 0.694 \\
Nondiabetics & $34(32)$ & \\
Postoperative antibiotics & $26(31)$ & \\
No postoperative antibiotics & $13(27)$ & \\
Location of abscess & & \\
$\quad$ Intersphincteric & $1(17)$ & \\
$\quad$ Ischiorectal & $4(40)$ & \\
$\quad$ Perianal & $33(29)$ & \\
$\quad$ Supralevator & $1(100)$ & \\
\hline
\end{tabular}

ization to the prescription or regimentation given in terms of agents, dosage, and duration. Antibiotics were prescribed based on intraoperative anecdotal findings of cellulitis and induration, not on any serum markers. In addition, patients were usually discharged before any cultures were reported to possibly tailor the antibiotics as per culture and sensitivity. 
Last, with regards to location of the initial abscess, it was postulated that deeper-seated abscesses would be more prone to fistulas due to incomplete drainage. However, that was not reflected in the data gathered. Two possible reasons for this were first due to most of the abscesses being perianal and not enough variety encountered to be representative for accurate analysis. Second, during the procedure itself, some of the surgeons may not accurately classify the abscesses, especially the junior residents to which most of the abscesses are delegated in Rashid Hospital.

This study performed has its inherent limitations due to the retrospective design and limited patient number. In addition, the follow-up of the patients was limited for many of the patients due to a large number of them being employed in manual labor and would not follow-up after wound healing. None of the risk factors included in our study were found to be statistically significant in the development of perianal fistulas after drainage of perianal abscesses.

\section{Acknowledgements}

The authors would like to acknowledge YT, general surgery residency director for his guidance and mentorship during all stages of the research.

\section{Statement of Ethics}

Ethical approval was obtained from the Dubai Scientific Research Ethics Committee with reference number DSREC/ RRP/2019/10 on 2019 May 9.

\section{Conflict of Interest Statement}

The authors have nothing to disclose.

\section{Funding Sources}

No funding was required or received for this research.

\section{Author Contributions}

All authors contributed equally in this paper.

\section{References}

1 Eisenhammer S. The anorectal fistulous abscess and fistula. Dis Colon Rectum. 1966 Mar-Apr;9(2):91-106.

2 Sugrue J, Nordenstam J, Abcarian H, Bartholomew A, Schwartz JL, Mellgren A, et al. Pathogenesis and persistence of cryptoglandular anal fistula: a systematic review. Tech Coloproctol. 2017;21(6):425-32.

3 Parks AG. Pathogenesis and treatment of fistuila-in-ano. Br Med J. 1961;1(5224):463-9.

4 Varut L, Hariruk Y, Darin. Incidence and factors influencing the development of fistulain-ano after incision and drainage of perianal abscesses. J Med Assoc Thai. 2010 Nov;93(1): 61-5.

5 Ali H, Philip IH, In-Lu AL, Maher AA. Who is at risk for developing chronic anal fistula or recurrent anal sepsis after initial perianal abscess? Dis of Colon and Rectum. 2009 Feb; 52(2):217-21.
6 Henrichsen S, Christiansen J. Incidence of fistula-in-ano complicating anorectal sepsis: a prospective study. Br J Surg. 1986;73(5):3712.

7 Wang D, Yang G, Qiu J, Song Y, Wang L, Gao $\mathrm{J}$, et al. Risk factors for anal fistula: a case-control study. Tech Coloproctol. 2014;18(7):6359.

8 De Lorenzi D. [Anorectal abscess and fistula]. Ther Umsch. 1997 Apr;54(4):197-201.

9 Sainio P. Fistula-in-ano in a defined population. Incidence and epidemiological aspects. Ann Chir Gynaecol. 1984;73(4):219-24.

10 Mazier WP. The treatment and care of anal fistulas: a study of 1,000 patients. Dis Colon Rectum. 1971 Mar-Apr;14(2):134-44.

11 Li-Hua Z, Ao-Zhe Z, Yu-Ying S, Xin L, Lan-Si J, Cong-Cong Z, et al. Impact of smoking on anal abscess and anal fistula diseases. Chinese Med J. 2018 May;131(9):1034-7.
12 Nunoo-Mensah JW, Balasubramaniam S, Wasserberg N, Artinyan A, Gonzalez-Ruiz C, Kaiser AM, et al. Fistula-in-ano: do antibiotics make a difference? Int J Colorectal Dis. 2006 Jul;21(5):441-3.

13 Ghahramani L, Minaie MR, Arasteh P, Hosseini SV, Izadpanah A, Bananzadeh AM, et al. Antibiotic therapy for prevention of fistula inano after incision and drainage of simple perianal abscess: a randomized single blind clinical trial. Surgery. 2017 Nov;162(5):1017-25.

14 Mocanu V, Dang JT, Ladak F, Tian C, Wang $\mathrm{H}$, Birch DW, et al. Antibiotic use in prevention of anal fistulas following incision and drainage of anorectal abscesses: a systematic review and meta-analysis. Am J Srg. 2019 May;217(5):910-7. 